\title{
Introducing Fundamental Microscopy Skills in a "Stage Wise" Manner to First Year Biology Students
}

\author{
Charlotte de Araujo, Karen J. Puddephatt, Gavin Ridgeway, Lynda H. \\ McCarthy, Andrew E. Laursen
}

\author{
Ryerson University, Department of Chemistry \& Biology, 350 Victoria St, Toronto ON M5B \\ 2K3 CAN \\ (cdearaujo@ryerson.ca; karenp@ryerson.ca; gridgeway@ryerson.ca; \\ 13mccart@ryerson.ca; alaursen@ryerson.ca)
}

\begin{abstract}
The microscope is an ubiquitous tool in the undergraduate biology laboratory. Implementing a hierarchical approach, students were introduced to skills in microscopy. Initially, students attempted to locate and examine a specimen on a prepared slide. Subsequently, students employed the microscope to recognize and describe the stages of mitosis in plant cells, observing particular features for each phase in onion (Allium) root tip. During the session, students created a wet mount with Brown planaria (Dugesia tigrina) and practiced using the microscope to observe live organisms. While developing skills in microscopy, parallel laboratory activities also included creating scientific illustrations and quantifying the proportion of cells in each stage of the cell cycle, integrating research skills (gathering, analyzing, interpreting data) as part of the exercise. In addition, we have adapted peer-to-peer teaching, where upper year students created a video microscopy tutorial. This supplemental resource provided first year students with an overview of the standard procedures and key components of a microscope. After reviewing the video, a majority of biology students felt more confident and comfortable using a microscope and more aware of practices which contribute to improper use. Coupling in-laboratory and online resources promoted student development of practical techniques.
\end{abstract}

Keywords: microscopy, mitosis, instructional video resources

Link to Original Poster File: https://doi.org/10.37590/able.v41.poster56

\section{Introduction}

Microscope are used for various purposes in the laboratory, including visualization and generation of images. This laboratory was designed to help students visualize the structure of cells and identify features of the stages of the cell cycle, incorporating conceptual material presented in lecture and to know how to identify different phases in mitosis. Here we use a tripartite approach to introduce first year biology students to the microscope in a 2.5 hour laboratory period. This involved: i) detailed laboratory manual which outlined instructions regarding microscope usage; ii) hands on practice in the laboratory, where students work in pairs; iii) supplementary instructional videos which provided guidelines and reviewed proper use of the in-house microscope. These strategies help target the diverse learning styles and needs of the Biology I students at Ryerson University.

While developing practical skills in microscopy, students are also required to complete in-laboratory report worksheets. Students practice making qualitative observations, gather data and gain experience creating hand-drawn visual representations of microscope images. In the laboratory, students also independently perform statistical calculations (mean, standard deviation) and also communicating their findings in a bar graph. This iterative approach builds on statistical concepts introduced in the previous laboratory (de Araujo et al. 2020, Laursen et al. 2018). 


\section{Student Outline \\ Microscopy: Cell Division}

\section{Objectives}

- To be able to properly use a microscope

- To be able to identify all parts of a microscope

- To be able to find and examine a specimen on a prepared slide

- To be able to describe and recognize the stages of mitosis in plant cells

- To be able to quantify and represent graphically the proportion of cells in each stage of the cell cycle.

- To practice use of your microscope for observing live organisms

- To observe the structures of the planaria

\section{Introduction: Microscopy using the Compound Light Microscope}

The compound light microscope uses light that is magnified and focused by two sets of lenses, the ocular (or eyepiece) and the objective. The total magnification of the specimen can be determined by multiplying the magnification of ocular and the objective together. The magnification of the ocular lens varies depending on the microscope. In our laboratories, our microscope ocular lenses have a magnification of 10x. The microscope has a number of different objective lenses called 4x (scanning power), 10x (low power), 40x (high power) and 100x (oil immersion).

Fill in Table 1.1 in the Reports Sheets at the end of this lab.

Illumination is given from a light source below the sample. Light will pass through clear areas on the slide and it will not pass through opaque areas. The lenses then magnify the image along with increasing image resolution. The resolving power of a microscope is its ability to distinguish between two objects that are very close together. The better the resolving power of the microscope, the better it is at distinguishing very small differences, and thus producing separate images of closely placed objects.

\section{Parts of a Microscope:}

Ocular Lenses: The ocular, or eyepiece, is the top lens system. It magnifies the image of the specimen. All the microscopes in the lab have two eyepieces and are known as binocular and their magnification power is 10x. There are some microscope models that have only one eyepiece, these are called monocular.

Objectives: The objective lenses magnify and resolve the image. As mentioned above, there are 4 different objective lens; 4x, 10x, 40x, and 100x.

Stage: The stage is the part of the microscope upon which the microscope slide rests. It is a platform, to which is a stage clamp is attached. These clamps hold the actual slide in place and while using the Stage Controls (in our case on the righthand side of the stage) the stage and thus the slide can be moved forwards and backwards as well as from left and right. The hole in the center of the stage allows light to come through from the light source below and illuminate the specimen on the slide

Coarse Adjustment: The coarse adjustment knob (outer knob on our microscopes) moves the stage up and down in course (large) increments. It allows the user to quickly get the specimen on the slide into the plane of focus and is used with the $4 \mathrm{x}$ objective. With this objective, the coarse adjustment knob is adequate for focusing.

Never use the course adjustment knob with the 40x or 100x objective lenses or you will damage the microscope and/or slide.

Once the slide is on the stage, always start with the stage all the way to the top and then move the stage away from the objective lens while observing the image though the ocular lens, using the course adjustment first. This way you can only move the stage down and away from the objective lens thus preventing damage to the microscope as well as the slide.

Fine Adjustment: The fine adjustment knob is used to move the stage up and down in very small increments. It is used with the higher magnification objective lenses (particularly the 40x or 100x objective) once the image is acquired, to obtain the final 'fine' focus of your object. On our microscopes, it is the inner knob.

Light: The light source is below the stage and the intensity is controlled (on our microscopes) with the red dial at base of the microscope on the left side. The light is focused through the condenser.

Condenser: Collects and focuses the light from the light source onto the specimen. It is located under the stage often in conjunction with an iris diaphragm.

Diaphragm: The diaphragm controls the amount of light that reaches the specimen by controlling the width of the light beam. 
It is located above the condenser and below the stage. Combined, the condenser and diaphragm control both the focus and quantity of light applied to the specimen.

\section{Using the Compound Light Microscope:}

1. Clean the lenses before and after using lens paper and lens cleaner.

2. Plug in microscope and turn the unit on using the switch on the base at the back.

3. Make sure that the $4 \mathrm{x}$ objective lens is in the working position.

4. The condenser should be fixed in position just below the stage.

5. With the stage in its lowest position, place a slide on the stage using the slide holder and using the stage adjustment knobs, move the slide so that the object to be viewed is under the objective lens.

6. Using the coarse adjustment knob, move the stage all the way to the top. Again, make sure the $4 \mathrm{x}$ objective is in position. If the 40x or 100x is in the working position, the slide will crash into the objective lens damaging both the slide and the objective lens.

7. Looking through the ocular lens, begin to move the coarse adjustment knob, lowering the stage until your specimen appears in focus.

8. Use the fine focus adjustment knob for precise focusing.

9. Swing the 10x objective lens into the working position. Do not move the stage! If further focusing is required, use ONLY the fine focus adjustment knob.

10. Repeat step 9 with the 40x objective. Since these microscopes are parfocal (no or minimal refocusing needed when changing between objectives) only fine adjustment will be needed at this point.

11. Move the mechanical stage all the way down. Remove your slide. If it was a prepared slide, remove oil with lens cleaner and lens paper and return it to your TA. Otherwise, dispose of your slide in the biohazard container.

12. Clean the oil immersion lens with lens cleaner and lens paper first. Using a clean piece of lens paper, clean the other lenses. Swing the $4 \mathrm{X}$ objective lens into the working position.

13. When done with the microscope for the day (and you are not done yet!), turn off the power and unplug the microscope and return it to its proper, labeled cupboard.

\section{Experiment 1.1b: Examining a Prepared Slide}

Obtain prepared slides (letter "e" and Cross Colored Threads, whole mount) from the side bench. Follow the procedure stated above, and examine the slide at 4x, 10x, and 40x. Start with the letter "e" slide. Once completed, repeat the steps using the Cross Colored Thread slide. The purpose of this exercise is to gain practice in using the microscope and finding your specimen.

Fill in Table 1.1 and answer the questions for Experiment 1.1b in the Reports Sheet found at the end of this lab. Sketch your observations in Figure 1.1.

\section{Experiment 1.2: Cell Division}

Now that you have had some guided experience using the microscope, in this exercise, you and your partner will apply this knowledge to find specific specimens under the microscope; in particular, the stages of mitosis in plant cells.

Mitosis: Nuclear division by mitosis results in two daughter cells which are genetically identical (barring mutation). This happens within a fertilized egg permitting it to grow into a multicellular organism. It is also a method to replace cells killed or removed in a wound. The only cells in humans that are not generally able to divide by mitosis are blood cells, muscle cells and neurons (there are some limited exceptions). Most have lost that ability when they differentiated (became specialized) to perform specific tasks. However, a current area of research explores de-differentiation of cells which would permit specialized cells to reacquire more stem-cell like properties, including cell division. Why might this be beneficial? Meiosis: Nuclear division results in daughter cells that are genetically different and that have half the number of chromosomes of the parent cell. These processes occur in reproductive cells (gametes) such as plant spores and animal eggs and sperm. In this laboratory, we will not observe meiotic division, but you should recognize that the stages of meiotic division have much in common and look much the same as stages in mitotic division, which you will observe in this laboratory.

Materials:

Prepared slide of Allium

\section{Exercise 1.2a: Mitosis in Plant Cells}


Use the compound light microscope to observe prepared slides of the onion (Allium) root tip. When looking at the slide, notice the rounded end which is the root cap. These cells have a relatively thin cytoplasm and are not dividing. Their function is simply protection of deeper tissue. The dividing cells will be just behind this cap in an area called the meristem, which is a specialized region of embryonic tissue. The cytoplasm of these cells is much denser and many dividing cells should be visible to you. The cells are not dividing in sequence, so consecutive stages will not be right next to each other.

1. Locate the following structures (not all will be visible in each cell):

cell wall nucleus cell plate nuclear membrane

chromosomes sister chromatids chromatin spindle apparatus

2. Locate a prophase cell.

i) How are the chromosomes arranged?

ii) What happens to the nuclear membrane?

iii) Do you see any spindle fibres?

3. Locate a metaphase cell.

i) How are the chromosomes arranged?

ii) What is the name of the portion of the cell where the chromosomes are arranged?

iii) How can you determine the poles of the cell?

4. Locate the anaphase cell.

i) Describe the position of the chromosomes.

ii) Even though it appears that the original number of the chromosomes has been reduced by half, this is not so. Each

daughter inherits the same number of chromosomes originally present in the parent.

5. Locate the telophase cell.

i) What criteria did you use to recognize this cell?

ii) What is happening to the nuclear envelope?

iii) How many nuclei should you see?

iv) Can you locate the cell plate?

6. Locate an interphase cell.

i) Are the chromosomes visible?

ii) How would you describe the appearance of the cell?

Note: Interphase is not part of mitosis. DNA, present in a granular-appearing mass known as chromatin, replicates. The nucleolus is present and the nuclear membrane is clearly visible.

Make drawings of each phase of mitosis in Figure 1.2 in the Reports Sheet found at the end of this lab, making note of the above questions with each phase of mitosis. These drawings can be started before coming to the lab so that you are familiar with the various stages of mitosis and therefore know what you should be looking for in each stage. Be prepared to identify these phases in the future, so make accurate detailed drawings of what you see. Complete Table 1.2 detailing the distinguishing characteristics that you were able to observe (do not simply list the characteristics given above, but complete this as notes that will be useful to you when asked to distinguish among cell stages in future).

\section{Exercise 1.2b: Quantifying the various stages of mitosis in Allium root tip}

Now that you are comfortable with identifying cells in different stages of the cell cycle (including mitosis), you will determine the number of cells in each stage.

1. Find an area of the root tip that is actively growing (has a large number of cells undergoing cell division). Scan using $4 \mathrm{x}$ or 10x objective.

2. Switch to the 40x objective. Within your field of view, count the total number of cells in each stage of the cell cycle (interphase, prophase, metaphase, anaphase, telophase). Record these observations in Table 1.3 in the Lab Report Sheet.

3. Move to a new field of view and repeat step 2 .

4. Move to a third field of view and repeat step 2.

5. Use the data recorded to calculate the frequencies of cells in each stage. Calculate the mean and standard deviation for your observations.

6. Use the calculated means and standard deviations to create bar graphs representing proportions of cells in each stage. Use the axes in Figure 1.3 for the purposes of creating your bar graph.

\section{Experiment 1.3: Observation of Planaria}

Next, you will practice using the microscope to observe the planaria. 
1. Transfer a planarian, along with a small volume of water, to a depression microscope slide. Gently lay a cover slip on the slide. The planarian will now be confined to the well of the depression slide and will be easier to observe.

2. Reduce the light intensity before placing the slide on the microscope stage! Remember that these organisms are highly light sensitive and photophobic. We want to limit the stress that will already be created by moving the organism to a slide. 3. Observe the planarian at low magnification. If you wish, you might move to higher magnification, but do not use any magnification greater than 100x (in other words, do not use any objective lens greater than 10x).

4. Draw the planarian in as much detail as you are able, particularly observe and draw the cerebral eyes. Sketch this in Figure 1.4 in your Laboratory Report Sheet.

\section{Laboratory Report Sheet}

\section{Experiment 1.1a: Microscopy using the Compound Microscope}

Table 1.1. Magnification for different ocular/objective lens combinations

\begin{tabular}{|l|l|l|l|}
\hline Lens & Lens Magnification & Ocular Magnification & Total Magnification \\
\hline Scanning power & & $10 \mathrm{x}$ & \\
\hline Low power & & $10 \mathrm{x}$ & \\
\hline High power & & $10 \mathrm{x}$ & \\
\hline Oil immersion & & $10 \mathrm{x}$ & \\
\hline
\end{tabular}

\section{Experiment 1.1b: Examining a Prepared Slide}

1. Sketch how you see the letter 'e' under the microscope and with the unaided eye
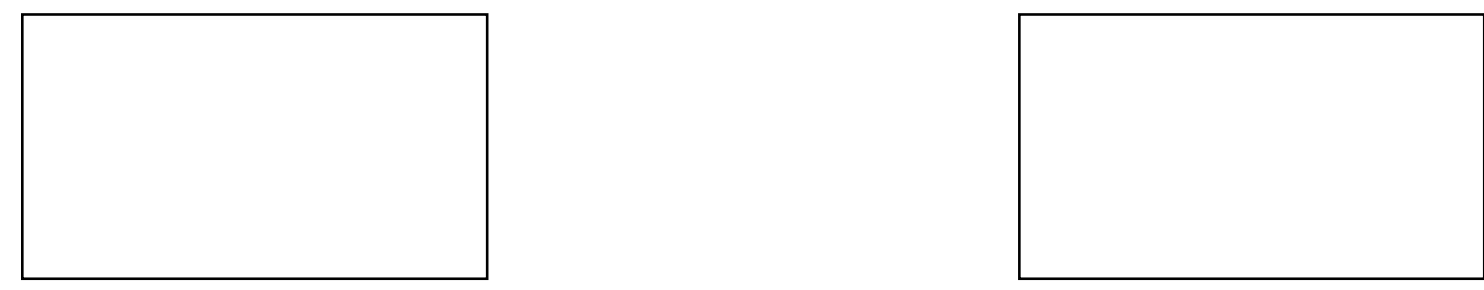

Figure 1.1. Image of letter 'e through microscope, (left) Image of letter 'e' as observed on the slide with naked eye (right)

1. On your particular slide of the threads, which thread was on top? in the middle? on the bottom?

2. What is meant by parfocal?

3. Why do we start with the $4 \mathrm{x}$ objective lens rather than with an objective with higher magnification?

4. Once the slide is on the stage, why do we always begin observation with the stage all the way up?

\section{Experiment 1.2: Cell Division - Exercise 1.2a, Mitosis in Plant Cells (Allium)}

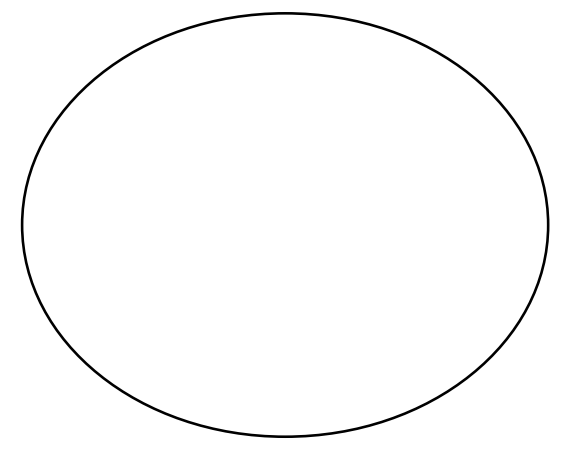

Figure 1.2a. Plant cell in Interphase

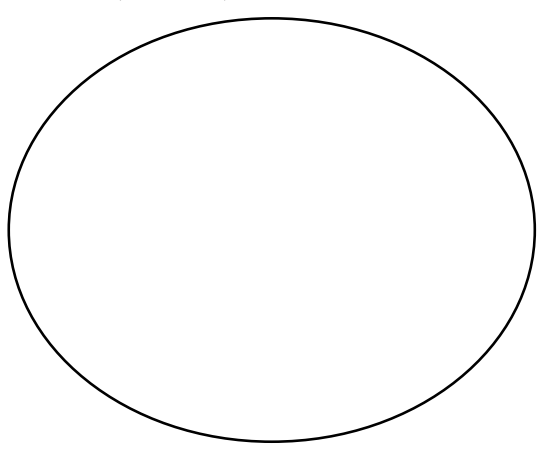

Figure 1.2b. Plant cell in Prophase 
Magnification:

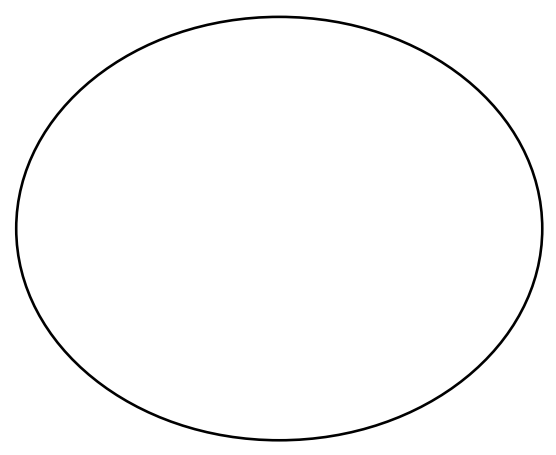

Figure 1.2c. Plant cell in Metaphase Magnification:

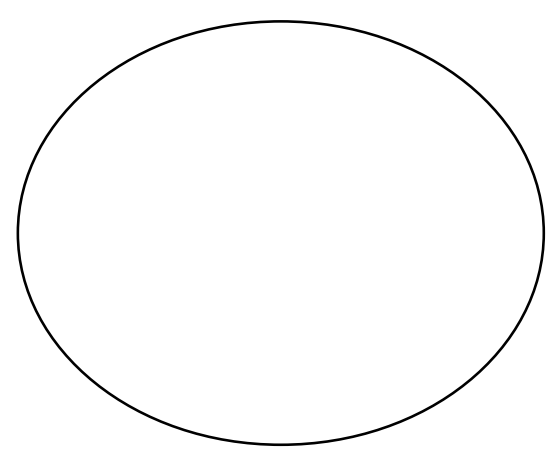

Figure 1.2e. Plant cell in Telophase

Magnification:
Magnification:

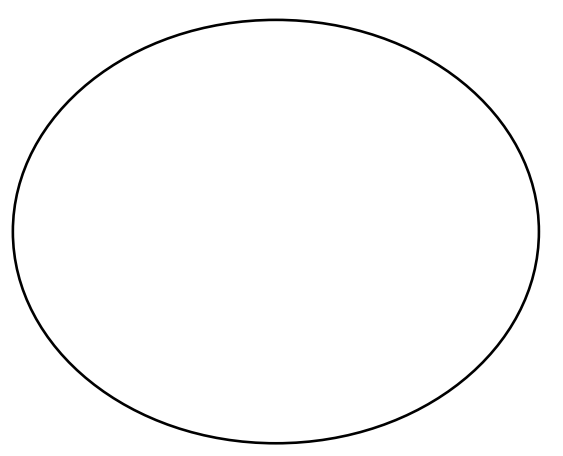

Figure 1.2d. Plant cell in Anaphase Magnification:

5. What is the distinguishing feature of each of the stages of mitosis, including interphase, in plant cells (as represented by Allium)? Answer this using Table 1.2

Table 1.2. Distinguishing characteristics of different stages of cell cycle.

\begin{tabular}{|l|l|}
\hline Phase & Distinguishing feature(s) in Allium (plant cells) \\
\hline Interphase & \\
\hline Prophase & \\
\hline Metaphase & \\
\hline Anaphase & \\
\hline Telophase & \\
\hline
\end{tabular}

Experiment 1.2: Cell Division - Exercise 1.2b, Quantifying the various stages of mitosis in Allium root tip 
6. Complete Table 1.3, recording your observations and calculations of cells in each stage of cell cycle. Use these to complete a bar graph representing your data (Fig. 1.3).

Table 1.3. Total number of cells within a field of view - each stage of cell cycle.

\begin{tabular}{|l|l|l|l|}
\hline Total number of cells & Field of View 1 & Field of View 2 & Field of View 3 \\
\hline Interphase & & & \\
\hline Prophase & & & \\
\hline Metaphase & & & \\
\hline Anaphase & & & \\
\hline Telophase & & & \\
\hline Proportion of cells & & & \\
\hline Interphase & & & \\
\hline Prophase & & & \\
\hline Metaphase & & & \\
\hline Anaphase & & & Standard Deviation \\
\hline Telophase & & Interphase & \\
\hline & Average Proportion & Prophase & \\
\hline Interphase & & Metaphase & \\
\hline Prophase & & Anaphase & \\
\hline Metaphase & & Telophase & \\
\hline Anaphase & & & \\
\hline Telophase & & & \\
\hline
\end{tabular}

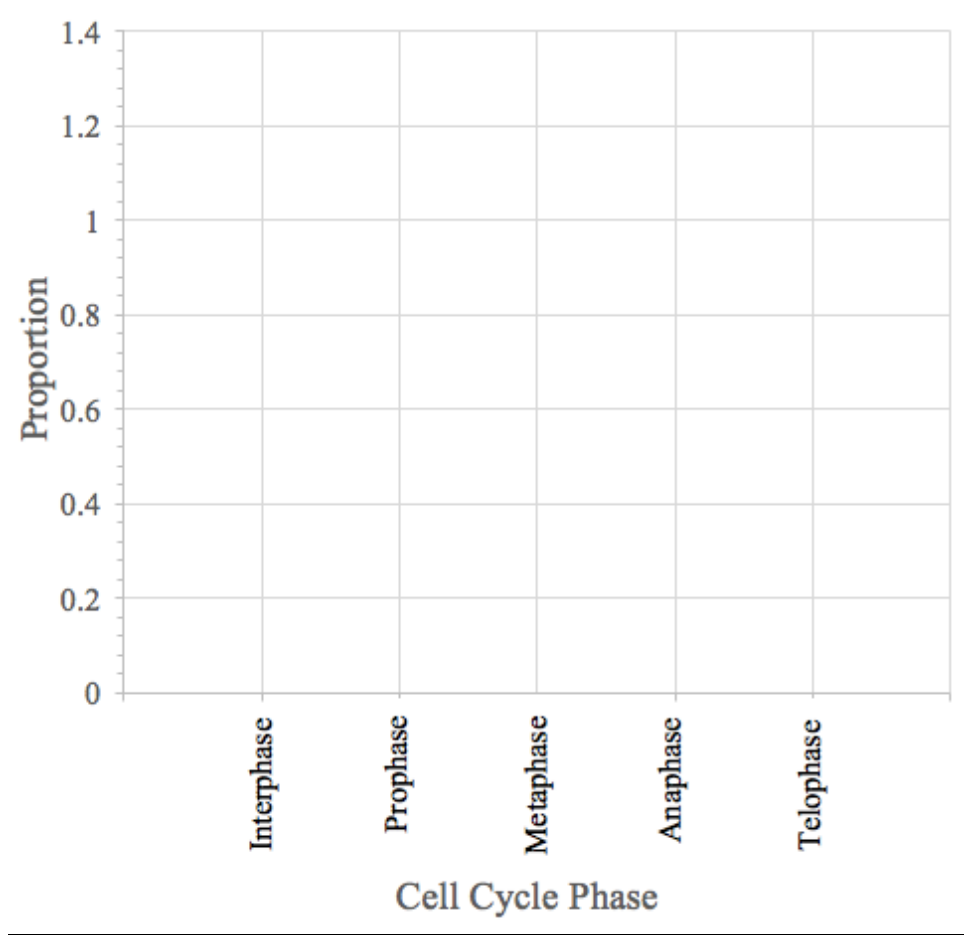

Figure 1.3. Proportion of cells in various stages of the cell cycle.

\section{Experiment 1.3: Observation of Planaria}


1. Sketch, with as much detail as possible, your planarian in Figure 1.4.

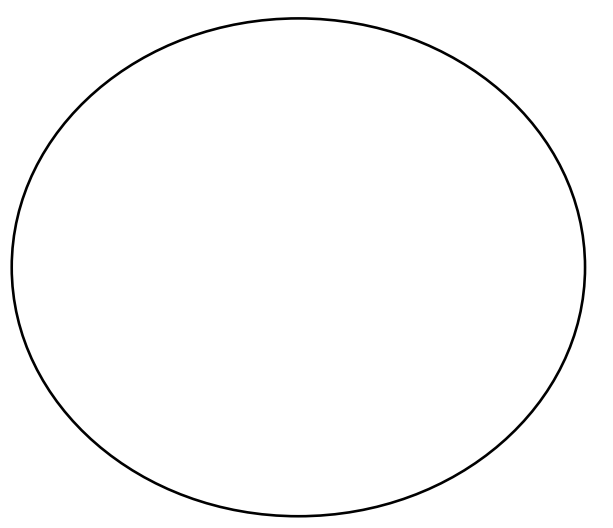

Figure 1.4. Planarian, Magnification: de Araujo, C, Pereira, N, Puddephatt, KJ, McCarthy, LH, Laursen, AE. 2020. Explaining the Scientific Method to First Year Biology Students in An Accessible Manner. Article 55 In: McMahon K, editor. Advances in biology laboratory education. Volume 41. Publication of the 41st Conference of the Association for Biology Laboratory Education (ABLE). https://doi.org/10.37590/able.v41.art56 


\section{Materials}

To operate this laboratory, we required the following materials: Leica Microscopes (Opti-Tech Scientific Inc), prepared slides of of letter 'e' (Ward's Science, Product code: 470177-692), Cross Colored Threads, whole mount (Ward's Science, Product code: 470176-898), Allilum root tip (Merlan Scientific, Product code: 302396). To create wet mounts, brown planaria (Merlan Scientific 132954), depression microscope slides (VWR 470235-728) and cover slips (VWR 470145-876) were used.

\section{Notes for the Instructor}

\section{Potential Considerations for the Practical Portion} Experiment 1.1b: Examining a Prepared Slide

Advise students to view the prepared slide without the aid of a microscope to ensure they observe how the image inverts when viewed using the microscope.

\section{Exercise 1.2a: Mitosis in Plant Cells}

While guiding students through this exercise remind students that unlike typical representations in their textbook, where cells line up neatly, they may be viewing cells from an angle.

\section{Safety Considerations}

Ensure students are aware about procedures in an instance of disposing slides or broken glass.

\section{Evaluation of Videos}

We incorporated two videos which featured laboratory techniques (how to use the microscope and how to create a wet mount) in addition to the practical component. When surveyed, the majority of Biology I students $(86.8 \%, \mathrm{n}=394)$ felt that the laboratory manual provides enough details regarding how to use a microscope. After viewing the video, $82.23 \%$ of the students felt more confident and comfortable with using a microscope in the laboratory. Many students $(82.49 \%)$ also agreed that after reviewing the video, they were more aware of practices which contribute to improper use of the microscope.

However, only $53.55 \%$ of the students felt the laboratory manual provided enough details to create a wet mount. After viewing the video, slightly more students (65.23\%) felt confident and comfortable with setting up a wet mount in the laboratory.

We would advise creating videos specific to the institution microscope tools. This imparts a greater value for the video, serving as a tool that can be used beyond the laboratory, concurrently aiding to train teaching assistants and also ensuring consistency in the curriculum across future course offerings.

\section{Cited References}

de Araujo C, Pereira N, Puddephatt KJ, McCarthy LH, Laursen AE. 2020. Explaining the scientific method to first year biology students in an accessible manner. Article 55, In: McMahon K, editor. Advances in biology laboratory teaching. Volume 41. Publication of the 41st Conference of the Association for Biology Laboratory Education (ABLE). https://doi.org/10.37590/able.v41.art56

Laursen A, McCarthy H, Puddephatt KJ, de Araujo C, Victorio-Walz L. 2018. Biology 143 Laboratory Manual. Plymouth, (MI): MacMillan Learning. p. 37-50.

\section{Acknowledgments}

We are grateful to the undergraduate class of Biology I (BLG 143) 2018 for their participation. Thanks to support from the Ryerson University Learning and Teaching Grant and Ryerson University Learning and Teaching Office.

\section{About the Authors}

Charlotte de Araujo, Ph.D. is an Assistant Professor in the Department of Chemistry and Biology, Ryerson University and an educator with The G. Raymond Chang School of Continuing Education.

Karen J. Puddephatt, M.Sc. is a laboratory technologist in the Department of Chemistry and Biology, at Ryerson University and CE Instructor.

Gavin Ridgeway, B.Com. is working on completing his undergraduate degree in biology during the course of this project.

Lynda H. McCarthy, Ph.D. is a Professor in the Department of Chemistry and Biology, Ryerson University and founder and Director of Ryerson Urban Water.

Andrew E. Laursen, Ph.D. is an Associate Professor in the Department of Chemistry and Biology, Ryerson University and Graduate Program Director for Environmental Applied Science and Management. 


\section{Mission, Review Process \& Disclaimer}

The Association for Biology Laboratory Education (ABLE) was founded in 1979 to promote information exchange among university and college educators actively concerned with teaching biology in a laboratory setting. The focus of ABLE is to improve the undergraduate biology laboratory experience by promoting the development and dissemination of interesting, innovative, and reliable laboratory exercises. For more information about ABLE, please visit http://www.ableweb.org/.

Advances in Biology Laboratory Education is the peer-reviewed publication of the conference of the Association for Biology Laboratory Education. Published articles and extended abstracts are evaluated and selected by a committee prior to presentation at the conference, peer-reviewed by participants at the conference, and edited by members of the ABLE Editorial Board. Published abstracts are evaluated and selected by a committee prior to presentation at the conference.

\section{Citing This Article}

de Araujo, C, Puddephatt, KJ, Ridgeway, G, McCarthy, LH, Laursen, AE. 2020. Introducing fundamental microscopy skills in a "stage wise" manner to first year biology students. Article 56 In: McMahon K, editor. Advances in biology laboratory education. Volume 41. Publication of the 41st Conference of the Association for Biology Laboratory Education (ABLE). https://doi.org/10.37590/able.v41.art56

Compilation (C) 2020 by the Association for Biology Laboratory Education, ISBN 1-890444-17-0. All rights reserved. No part of this publication may be reproduced, stored in a retrieval system, or transmitted, in any form or by any means, electronic, mechanical, photocopying, recording, or otherwise, without the prior written permission of the copyright owner.

ABLE strongly encourages individuals to use the exercises in this volume in their teaching program. If this exercise is used solely at one's own institution with no intent for profit, it is excluded from the preceding copyright restriction, unless otherwise noted on the copyright notice of the individual chapter in this volume. Proper credit to this publication must be included in your laboratory outline for each use; a sample citation is given above. 